\title{
Muscle-specific GSTM2-2 on the luminal side of the sarcoplasmic reticulum modifies RyR ion channel activity
}

\author{
Lan Wei ${ }^{1}$, Yasser A. Abdellatif ${ }^{1,2}$, Dan Liu ${ }^{1}$, Takashi Kimura ${ }^{3}$, Marjorie Coggan, \\ Esther M. Gallant, Nicole A. Beard, Philip G. Board ${ }^{4}$, Angela F. Dulhunty*,4 \\ Division of Molecular Bioscience, John Curtin School of Medical Research, Australian National University, \\ PO Box 334, Canberra City, ACT 2601, Australia
}

Received 6 September 2007; received in revised form 30 November 2007; accepted 18 December 2007

Available online 20 January 2008

\begin{abstract}
We show that a glutathione transferase (GST) protein, which is recognised by an antibody against the muscle-specific human GSTM2-2 (hGSTM2-2), is associated with the lumen of the sarcoplasmic reticulum (SR) of cardiac muscle, but not skeletal muscle. We further show that hGSTM2-2 modifies both cardiac and skeletal ryanodine receptor (RyR) activity when it binds to the luminal domain of the RyR channel complex. The properties of hGSTM2-2 were compared with those of the calsequestrin (CSQ), a $\mathrm{Ca}^{2+}$ binding protein also present in the lumen of the SR which, like GSTM2-2, contains a thioredoxin-fold structure and modifies RyR activity (Wei, L., Varsanyi, M., Dulhunty, A. F., Beard, N. A. (2006). The Biophysical Journal, 91, 1288-1301). The glutathione transferase activity of hGSTM2-2 is strong, while CSQ is essentially inactive. Conversely CSQ is a strong $\mathrm{Ca}^{2+}$ binder, but hGSTM2-2 is not. The effects of luminal hGSTM2-2 on RyR activity differ from those of CSQ in that hGSTM2-2 activates RyRs by increasing their open probability and conductance and the effects are independent of luminal $\mathrm{Ca}^{2+}$ concentration. The results suggest that GSTM2-2 can interact with specific luminal sites on the RyR complex and that the interaction is likely to be within the pore of the RyR channel. The differences between the effects of CSQ and hGSTM2-2 suggest that the thioredoxin fold is not a major determinant of the luminal actions of either protein. The results indicate that GSTM2-2 is a novel luminal regulator of the RyR channels in the heart.
\end{abstract}

(C) 2008 Elsevier Ltd. All rights reserved.

Keywords: Ryanodine receptor; Mu class glutathione transferase; Sarcoplasmic reticulum lumen; Cardiac muscle; Skeletal muscle; Muscle calcium signaling

\footnotetext{
* Corresponding author. Tel.: +61 26125 4491; fax: +6126125 4761 .

E-mail address: angela.dulhunty@anu.edu.au (A.F. Dulhunty).

1 Authors made equal contributions to the project.

2 Present address: Institute of Cardiovascular Sciences, St. Boniface General Hospital Research Centre, University of Manitoba, Winnipeg, Canada R2H2A6.

${ }^{3}$ Present address: Department of Neurology and Stroke, Hyogo College of Medicine, 1-1 Mukogawa-cho Nishinomiya, HYOGO 663-8501, Japan.

${ }^{4}$ Authors made equal senior contributions to this project.
}

\section{Introduction}

The ryanodine receptor (RyR) ion channel is responsible for releasing $\mathrm{Ca}^{2+}$ from the sarcoplasmic reticulum (SR) in cardiac and skeletal muscle and is essential for muscle contraction. The RyR forms a massive protein complex that is regulated by multiple factors on both the cytoplasmic and the luminal side of SR membrane. Its regulation by cytoplasmic factors has been investigated extensively, but luminal factors are less well defined. In this study we examine the luminal regulation of the 
RyR by the glutathione transferase (GST) GSTM2-2, which is enriched in the cytoplasm of skeletal and cardiac muscle (Abdellatif et al., 2007). Proteins in the GST structural family have diverse actions in addition to their well recognised role as phase II detoxifying enzymes (Board et al., 2000). We have found that several members of the GST family, including GSTM2-2 regulate RyR channels when they bind to the cytoplasmic domain of the RyR complex (Board, Coggan, Watson, Gage, \& Dulhunty, 2004; Dulhunty, Gage, Curtis, Chelvanayagam, \& Board, 2001; Dulhunty, Pouliquin, Coggan, Gage, \& Board, 2005). In a recent study, human GSTM2-2 (hGSTM2-2) was added to the luminal side of RyR channels in lipid bilayers as a negative control for the action of the protein on the cytoplasmic side of the channel. Surprisingly hGSTM2-2 in the luminal solution did alter RyR activity, in a manner which differed from its cytoplasmic effects.

We report here that a soluble protein, detected by an antibody to hGSTM2-2, is present within the lumen of the SR of cardiac, but not skeletal, muscle. The GST class of proteins contains a thioredoxin fold with a characteristic mix of $\beta$-strands and $\alpha$-helices in the $\mathrm{N}$ terminal domain and $\alpha$-helices in the $\mathrm{C}$-terminal domain. Thioredoxin-fold structures also occur in calsequestrin (CSQ), the $\mathrm{Ca}^{2+}$ binding protein in the SR (Wang et al., 1998). CSQ in the luminal solution modifies RyR channels either by binding to the proteins triadin and junctin that span the SR membrane and anchor CSQ to the RyR (Beard, Laver, \& Dulhunty, 2004; Wei, Varsanyi, Dulhunty, \& Beard, 2006) or by binding directly to the RyR protein (Herzog, Szegedi, Jona, Herberg, \& Varsanyi, 2000; Szegedi, Sarkozi, Herzog, Jona, \& Varsanyi, 1999). We reasoned that there may be a functional overlap between CSQ and GSTM2-2 in the lumen of the SR and that it was possible that both proteins may bind to the same site on the RyR through their thioredoxin folds. Therefore, we have compared several properties of hGSTM2-2 and CSQ, including their actions on RyR channel activity.

\section{Materials and methods}

\subsection{SR vesicle preparation}

Cardiac SR vesicles (CP4) were prepared from sheep heart (Chamberlain \& Fleischer, 1988; Laver et al., 1995). SR vesicles were prepared from back and leg muscle from New Zealand white rabbits. Crude skeletal muscle preparations, SP3 (Saito, Seiler, Chu, \& Fleischer, 1984) and LP3 (Kim, Ohnishi, \& Ikemoto, 1983), were used. SP3 vesicles were run on a sucrose gradient to concentrate heavy SR, SB4 (Ahern, Junankar, \& Dulhunty, 1994; Saito et al., 1984).

\subsection{CSQ and hGSTM2-2 purification}

CSQ1 was purified from skeletal muscle as described previously (Wei et al., 2006). Sheep cardiac CSQ2 was prepared by elution from vertical slab SDSpolyacrylamide gels (Hoefer SE 600 electrophoresis system). Solubilised cardiac SR was separated on 8.5\% SDS polyacrylamide gels with Laemmli buffer (Laemmli, 1970). The protein standards were excised, copper stained (Bio-Rad) and aligned with the gel to locate CSQ which was excised, immersed in $20 \mathrm{mM}$ MOPS, $150 \mathrm{mM} \mathrm{NaCl}, 200 \mu \mathrm{M}$ EGTA, $0.2 \%$ Triton X$100, \mathrm{pH} 7.4$ and incubated at $37^{\circ} \mathrm{C}$ for $12-24 \mathrm{~h}$. Eluted CSQ2 was dialysed against the same buffer without Triton X-100 and stored at $-20^{\circ} \mathrm{C}$. Recombinant CSQ1 was expressed and purified (Beard et al., 2005).

Recombinant GSTM2-2 was prepared according to (Ross \& Board, 1993) with some modifications. An E. Coli (TG1) clone containing the hGSTM2-2 expression plasmid was grown to an OD555 of $\sim 0.6$ in $500 \mathrm{ml}$ of liquid broth culture media containing $0.1 \mathrm{mg} / \mathrm{ml}$ ampicillin. GSTM2-2 expression was induced with $1 \mathrm{mM}$ isopropyl$\beta$-D-thiogalactopyranoside and the culture grown for at least $4 \mathrm{~h}$ at $37^{\circ} \mathrm{C}$. Bacteria were collected by centrifugation at $2000 \times g$ for $20 \mathrm{~min}$. The pellet was dispersed by mild sonication for $20 \mathrm{~s}$ in a buffer containing $20 \mathrm{mM}$ Tris, $\mathrm{pH} 7.3$ and $0.1 \mathrm{mg} / \mathrm{ml}$ lysozyme and lysed on ice for $1 \mathrm{~h}$. The lysate was further sonicated and then centrifuged at $20000 \times g$ for $20 \mathrm{~min}$. The supernatant was collected and incubated with glutathione agarose beads overnight at $4{ }^{\circ} \mathrm{C}$ with gentle rotation. The beads were then centrifuged at $805 \times g$ for $5 \mathrm{~min}$ and washed twice with $20 \mathrm{mM}$ Tris, $\mathrm{pH}$ 7.3. Bound GST was eluted with $20 \mathrm{mM}$ Tris, $\mathrm{pH} 9.6$ and $10 \mathrm{mM}$ GSH. The eluted protein was assayed for activity using 1,chloro-2,4-dinitrobenzene and purity was assessed by SDS $12.5 \%$ polyacrylamide gel electrophoresis. Consistent results were obtained using at least 10 different samples of hGSTM2-2.

\subsection{Detection of luminal GSTM2-2}

Skeletal (SB4, SP3 and LP3) or cardiac (CP4) SR vesicles (each $1.5 \mathrm{mg}$ protein) were washed in $2 \mathrm{ml}$ of storage buffer (50 mM HEPES pH 7.4, $150 \mathrm{mM} \mathrm{KCl}$, $1 \mathrm{mMCaCl}_{2}$, protease inhibitors) to remove soluble GST in the solution or on the cytoplasmic surface of the vesicles. The vesicles were pelleted by centrifugation at $100000 \times g$ for $20 \mathrm{~min}$. The supernatant (S1) was concentrated to $100 \mu \mathrm{l}$ and the pellet (P1) was resuspended 




Fig. 1. Detection of soluble hGSTM2-2-like protein in the lumen of cardiac SR. Procedures are described, and nomenclature defined, in Section 2. (A) Cardiac CP4 vesicles, (B) skeletal SB4 vesicles. In (A) and (B): lane 1, washed parent SR vesicle (W); lane 2, supernatant (S1) after centrifugation of the washed sample; lane 3, re-suspended P1 vesicles pelleted after washing; lane 4, S2 supernatant after solubilisation; lane 5, insoluble P2 fraction after solubilisation; lane 6, hGSTM2$2(0.5 \mu \mathrm{g})$ added to S2; lane 7, hGSTM2-2 $(0.5 \mu \mathrm{g})$ alone. Similar amounts of material were compared in the skeletal and cardiac preparations. Starting material contained $1.5 \mathrm{mg}$ of protein and wash volumes, concentration steps and volumes $(20 \mu \mathrm{l})$ added in each lane were identical. (C) Cross-reactivity of polyclonal hGSTM2-2 antiserum. Panel (i), western blot of human GSTs with rabbit anti-hGSTM2-2 serum. Panel (ii), human GSTs from an identical SDS PAGE gel stained with Coomassie blue. Track 1, molecular size markers; Track 2, GSTA1; Track 3, GSTA3; Track 4, GSTM1; Track 5, GSTM2; Track 6, GSTP1; Track 7, GSTZ1.

in $500 \mu$ l of buffer. The P1 vesicles were then solubilised by Triton X-100 $(0.5 \%)$, on ice for $30 \mathrm{~min}$ and centrifuged (100 $000 \times g$ for $20 \mathrm{~min})$. The supernatant (S2) containing soluble luminal protein was concentrated to $100 \mu \mathrm{l}$ and the P2 pellet (containing insoluble membrane fragments) was resuspended in $100 \mu$ l of storage buffer. Samples in Fig. 1 are the parent vesicles CP4 or SB4 (W) and the S1, P1, S2 and P2 fractions from CP4 (1A) and SB4 (1B). $20 \mu$ l of each sample was loaded on $10 \%$ SDS-PAGE. Proteins were blotted onto nitrocellulose membrane and probed using the polyclonal antibody raised in rabbit against GST-4 purified from human skeletal muscle (Board, Suzuki, \& Shaw, 1988). GST-4 is now known as GSTM2-2 (Mannervik et al., 1992).

\section{4. $\mathrm{Ca}^{2+}$ binding properties}

Proteins were probed with the cationic carbocyanine dye "stains all" (Campbell, MacLennan, \& Jorgensen, 1983) or ${ }^{45} \mathrm{Ca}^{2+}$ (Maruyama, Mikawa, \& Ebashi, 1984).

\subsection{Glutathione transferase activity}

GST activity was measured using the substrate 1-chloro-2,4-dinitrobenzene (CDNB) (Ross \& Board, 1993). Reaction rates were corrected for non-enzymatic activity with the buffer system alone.

\subsection{Single channel recording and analysis}

Lipid bilayers techniques and single channel analysis have been described previously (Ahern et al., 1994; Laver et al., 1995; Wei et al., 2006).

\subsection{Statistics}

Average data are given as mean \pm S.E.M. Difference between values was evaluated by Student's $t$-test. Differences were considered significant with $P \leq 0.05$.

\section{Results}

\subsection{GSTM2-2-like protein in the lumen of cardiac $S R$}

A soluble hGSTM2-2-like protein was detected in the cytoplasm of rabbit skeletal and sheep cardiac muscle fibres (Abdellatif et al., 2007), but its compartmentalisation into the lumen of the SR has not been explored. In 2 identical experiments, anti-hGSTM2-2 antibody detected a protein with the same molecular mass as hGSTM2-2 in the cardiac CP4 vesicles (Fig. 1A, lane 1, W), but not skeletal SB4 vesicles (Fig. 1B, lane 1, W). The protein remained associated with the cardiac vesicle (P1) pellet after washing, but not the wash solution (S1) (Fig. 1A, lanes 2 and 3), indicating that it was located on the SR membrane or within the SR vesicles. The proteins released by solubilisation of the SR vesicles were probed. The hGSTM2-2 antibody detected a protein in the supernatant (S2, containing soluble luminal proteins (Fig. 1A, lane 4)), but not with the membrane pellet (P2, Fig. 1A, lane 5) from cardiac fractions. Antibody specificity showed cross-reaction with GSTM2-2 and the Mu class isoenzyme GSTM1-1 but not with Alpha, Pi, or Zeta classes (Fig. 1C). GSTM1-1 has $99.1 \%$ identity with GSTM2-2 and is expressed in human skeletal 
muscle and heart, in smaller quantities than GSTM2-2 (Board et al., 1988). The mobility difference between proteins in lanes 3 and 4 (Fig. 1A) is due to the amounts of proteins loaded ( $12 \mu \mathrm{g}$ in lane 3 and $300 \mu \mathrm{g}$ in lane 4$)$. Similar retardation occurred when additional recombinant hGSTM2-2 was added to the soluble luminal sample (Fig. 1A and B, lane 6) or run alone (Fig. 1A and B, lane 7).

Less purified skeletal SP3 and LP3 preparations were compared with SB4 to test the possibilities (a) that a GSTM2-2-like protein had been leached from the SB4 vesicles in the sucrose gradient and (b) that CP4 may have contained outside-out plasmalemma vesicles holding cytoplasmic GSTM2-2. The results (not shown) were identical to those of SB4 showing that a soluble GSTM22-like protein is located within the cardiac SR.

\subsection{Thioredoxin folds in CSQ and GSTM2-2}

A comparison of the structure of each of the three thioredoxin-fold domains of CSQ with the thioredoxin domain of hGSTM2-2 shows greatest similarity with the CSQ N-terminal domain I and central domain II (Fig. 2). A vector alignment search tool (VAST; http:// www.ncbi.nlm.nih.gov/Structure/VAST/vast.shtml)

indicated $22 \%$ sequence identity with domain I, $2.2 \%$ identity with domain II, but no identity with domain III. The VAST scores for structural similarity between hGSTM2-2 and CSQ were 6.3 for domain I, 7.6 for domain II and 0 for domain III, with $P$ values of 0.047 and 0.0006 , respectively for domains I and II, indicating a low probability that the structural similarities occurred by chance. The $22 \%$ sequence similarity between CSQ domain I and hGSTM2-2 is equivalent to the similarity between GSTM2-2 and CLIC1 (chloride intracellular channel) which belongs to the GST structural family (Dulhunty et al., 2001). Since domain III of CSQ contains the major $\mathrm{Ca}^{2+}$ binding motif (Wang et al., 1998), the lack of sequence and structural similarity with hGSTM2-2 suggests that the $\mathrm{Ca}^{2+}$ binding properties of the proteins may differ. In addition, since CSQ lacks typical GST active site residues it might, as with some other members of the GST structural family (Board et al., 2004), lack glutathione transferase activity.

\section{3. $\mathrm{Ca}^{2+}$ binding capacity and glutathione transferase activity}

CSQ contains surfaces of negative charge density which may form $\mathrm{Ca}^{2+}$ binding sites (Wang et al., 1998). The surface of the hGSTM2-2 monomer also shows regions of high negative charge density (Fig. 3A-D) that
(A)
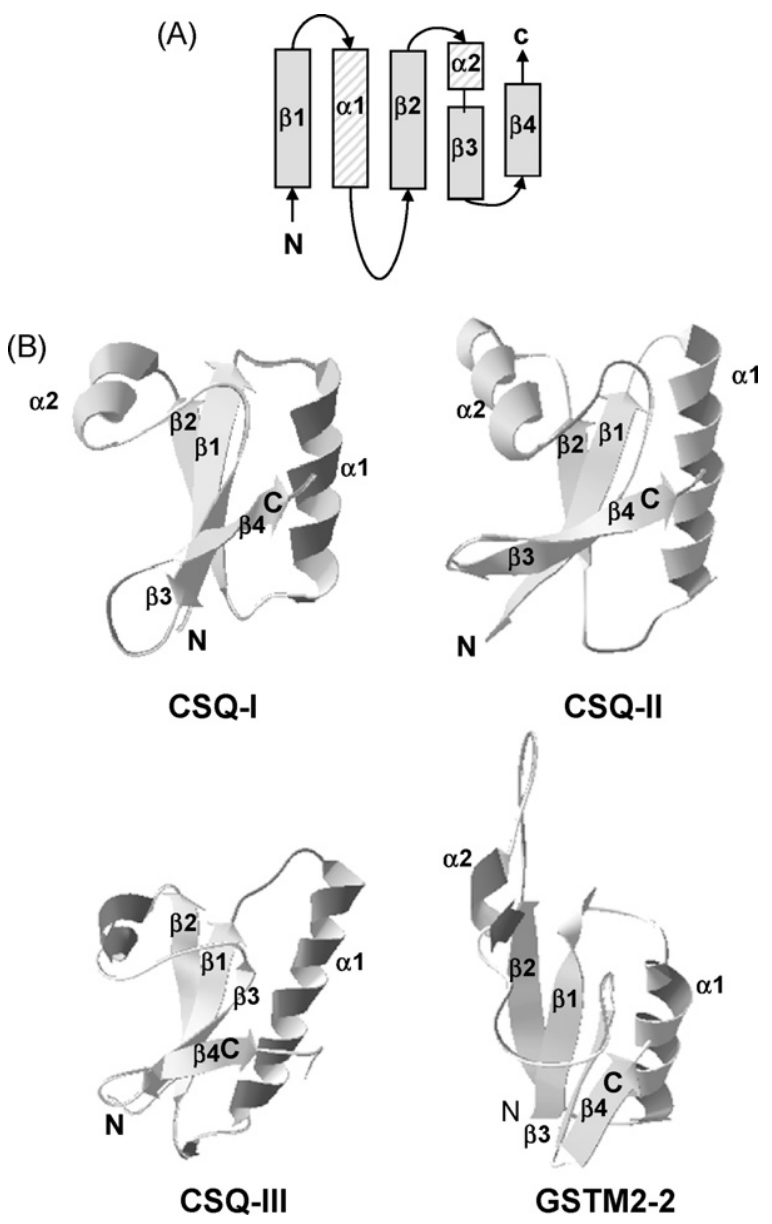

Fig. 2. Thioredoxin folds of CSQ and hGSTM2-2. (A) Line diagram showing common elements of thioredoxin folds. (B) The three CSQ thioredoxin-fold motifs (the N-terminal CSQ1, CSQ2, and the C-terminal CSQ3) in rabbit skeletal CSQ (PDB1A8Y) and the hGSTM2-2 monomer (PDB3GTU). The amino and carboxy terminals are labelled $\mathrm{N}$ and $\mathrm{C}$. The carboxy terminal strand is hidden by the $\alpha 1$-helix in most cases, therefore the C-terminal end of the $\beta 4$ strand contains the C label for clarity. Figures generated using Swiss Pdb viewer (http://www.expassy.org.spdbv/).

could facilitate $\mathrm{Ca}^{2+}$ binding. The $\mathrm{Ca}^{2+}$ binding ability of hGSTM2-2 was compared with that of rabbit skeletal muscle CSQ (CSQ1) using the anionic dye "stains-all", which develops a strong blue colour with proteins that are rich in acidic residues. The intensity of blue staining of CSQ1 after $2 \mathrm{~h}$ exposure to the dye reflects its high acidic residue content and is indicative of its high $\mathrm{Ca}^{2+}$ binding capacity (Fig. 3E, lane 1). The violet colour of hGSTM22 monomer after $12 \mathrm{~h}$ exposure (Fig. 3E, lane 2), reflects lower acidic residue content and indicates weak $\mathrm{Ca}^{2+}$ binding that was however greater than acidic content of serum albumin (pink colour after $24 \mathrm{~h}-$ Fig. 3E, lane 3 ). In ${ }^{45} \mathrm{Ca}$ overlay, equivalent amounts of hGSTM2- 
2 and CSQ1 were electrophoresed on a $10 \%$ native polyacrylamide gel (Fig. 3F). Autoradiographs showed strong $\mathrm{Ca}^{2+}$ binding to CSQ after $12 \mathrm{~h}$ exposure to ${ }^{45} \mathrm{Ca}$ and weaker binding to the hGSTM2-2 dimer after $27 \mathrm{~h}$ (Fig. 3G).
Glutathione transferase activity was determined from the rate of conversion of CDNB to its thioether with GSH. The activity was 100 -fold-1000-fold higher with hGSTM2-2 than with CSQ1, with either $50 \mu$ M or $1 \mathrm{mM}$ $\mathrm{Ca}^{2+}$ (Table 1). The levels of activity with CSQ were
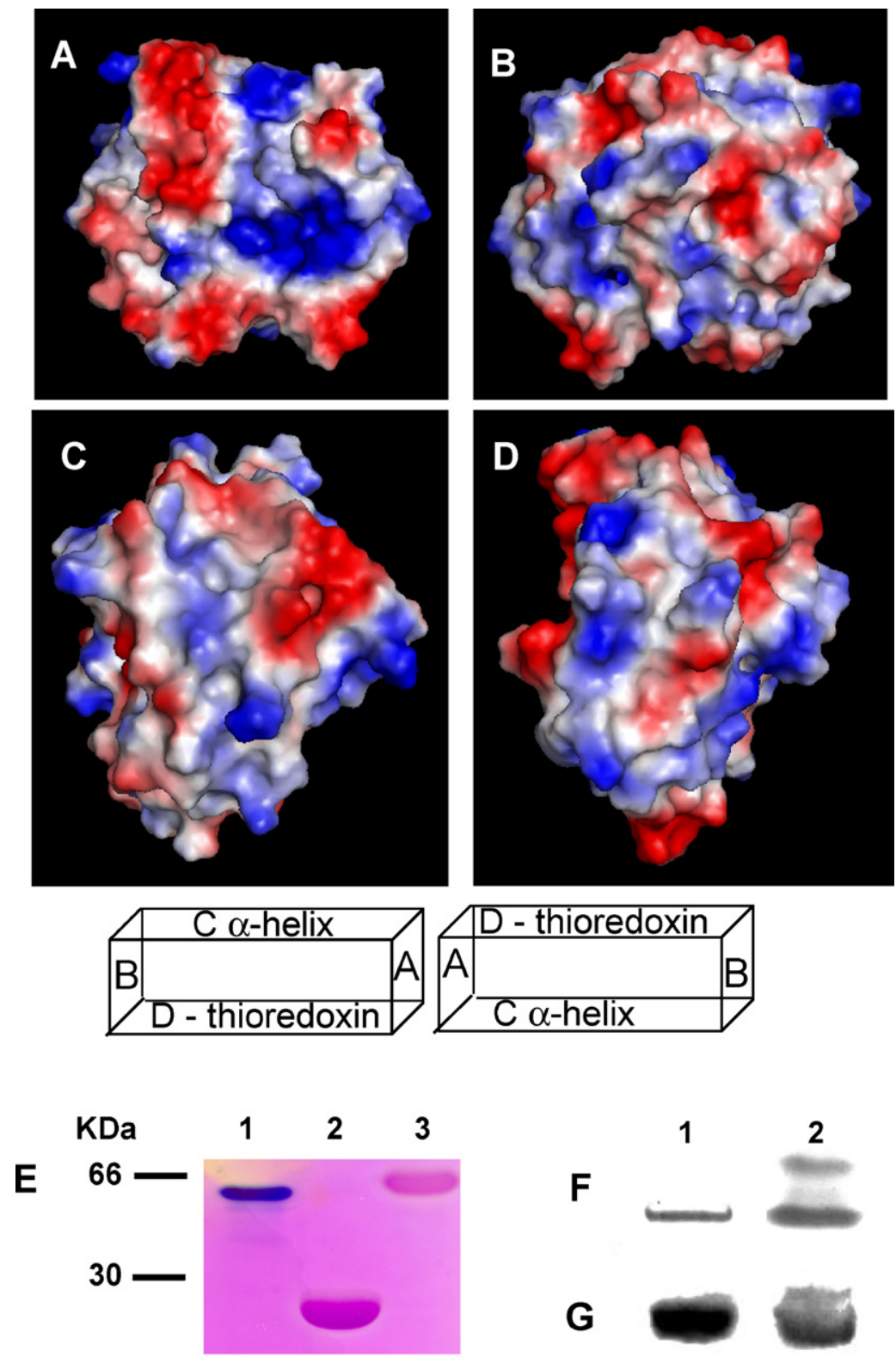

Fig. 3. $\mathrm{Ca}^{2+}$ binding capacity of hGSTM2-2. (A)-(D) Distribution of positive (blue) and negative (red) charge densities on the surfaces of the hGSTM2-2 dimer ((Patskovska, Fedorov, Patskovsky, Almo, \& Listowsky, 1998) PDB \# 1XW5) using the program *http://www.pymol.org* (published by DeLano Scientific). The distribution of charges in en face views of the: (A) dimer interface surface; (B) opposite surface, parallel to the dimer interface; (C) $\alpha$-helical domain; (D) thioredoxin domain. Schematic surfaces in A-D. (E) 12.5\% SDS-PAGE gel with "Stains-all": lane 1, CSQ $(12 \mu \mathrm{g})$; lane 2, hGSTM2-2 monomer $(30 \mu \mathrm{g})$; lane 3, bovine serum albumin (30 $\mu \mathrm{g})$. Markers are 66 and $30 \mathrm{kDa}$. (F) Coomassie Blue staining of a 10\% native gel showing CSQ monomer (lane 1) and hGSTM2-2 dimer (lane 2) running at similar positions. (G) ${ }^{45} \mathrm{Ca}$ overlay of equivalent amounts of CSQ monomer (lane 1) and hGSTM2-2 dimer (lane 2) on a $10 \%$ native gel. The CSQ band was observed after a $12 \mathrm{~h}$ exposure to ${ }^{45} \mathrm{Ca}$, while the weaker hGSTM2-2 band observed after $27 \mathrm{~h}$. 
Table 1

Glutathione transferase activity (DCNB as a substrate)

\begin{tabular}{llll}
\hline & \multicolumn{2}{l}{ Activity (nM/mg/min) } & \\
\cline { 2 - 4 } & Recombinant hGSTM2-2 & Rabbit skeletal muscle CSQ & Recombinant CSQ \\
\hline $50 \mathrm{M} \mathrm{Ca}^{2+}$ & $29.41 \pm 1.90(3)$ & $0.36 \pm 0.01 *(3)$ & $0.031 \pm 0.03 * \#(3)$ \\
$1 \mathrm{mM} \mathrm{Ca}^{2+}$ & $31.71 \pm 0.49(4)$ & $0.40 \pm 0.04 *(4)$ & $0.04 \pm 0.03 * \#(4)$ \\
\hline
\end{tabular}

The asterisks (*) indicates CSQ1 values significantly different from hGSTM2-2, the hash (\#) indicates significant differences between recombinant and skeletal muscle CSQ1. Average data is mean \pm S.D. Numbers of observations are in parentheses.

very low. The differences between the native and recombinant CSQ were significant, but small compared with the greater hGSTM2-2 activity and were not investigated further.

\subsection{Effects of hGSTM2-2 added to the luminal side of RyRs with $1 \mathrm{mM}$ luminal $\mathrm{Ca}^{2+}$}

RyRs incorporated into bilayers with $1 \mathrm{mM} \mathrm{Ca}^{2+}$ in the luminal (trans) solution retain normal triadin and junctin bound to RyR1 and the CSQ polymer, indicated by electrophysiological measurements, binding assays and chemical cross-linking (Beard, Sakowska, Dulhunty, \& Laver, 2002; Wei et al., 2006). Adding additional CSQ to the luminal solution does not alter RyR activity, presumably because the CSQ binding sites on triadin and junctin are occupied by endogenous CSQ (Beard et al., 2002). In contrast, adding hGSTM2-2 under these conditions increased cardiac RyR2 activity (Fig. 4A), indicating that the interaction site on RyR2 for hGSTM22 differed from the CSQ binding site. The increase in channel conductance with hGSTM2-2 (Fig. 4A) is addressed below. Luminal hGSTM2-2 $\geq 8 \mu \mathrm{M}$ maximally increased open probability $\left(P_{\mathrm{o}}\right)$ and conductance; concentrations between 8 and $30 \mu \mathrm{M}$ were routinely used.

Open probabilities $\left(P_{\mathrm{o}}\right)$ under control conditions are given in Table 2. The particular control condition was established at the start of the experiment after vesicle incorporation and perfusion of the "incorporation" solu-

\section{Table 2}

Values of $P_{\mathrm{o}}$ of RyR1 and RyR2 under control conditions with various cytoplasmic (cis) and luminal (trans) $\left[\mathrm{Ca}^{2+}\right]$ used in the experiments described in Figs. 4 and 5

\begin{tabular}{lllll}
\hline & Cis $\mathrm{Ca}^{2+}$ & Trans $\mathrm{Ca}^{2+}$ & Mean \pm S.E.M. & $n$ \\
\hline RyR1 & $100 \mathrm{nM}$ & $1 \mathrm{mM}$ & $0.014 \pm 0.006$ & 12 \\
RyR1 & $500 \mathrm{nM}$ & $100 \mathrm{nM}$ & $0.018 \pm 0.009$ & 26 \\
RyR1 & $100 \mu \mathrm{M}$ & $1 \mathrm{mM}$ & $0.060 \pm 0.012$ & 8 \\
RyR2 & $500 \mathrm{nM}$ & $1 \mathrm{mM}$ & $0.032 \pm 0.018$ & 24 \\
RyR2 & $500 \mathrm{nM}$ & $100 \mathrm{nM}$ & $0.091 \pm 0.063$ & 24 \\
RyR2 & $100 \mu M$ & $100 \mathrm{nM}$ & $0.545 \pm 0.054$ & 6 \\
\hline
\end{tabular}

(A)

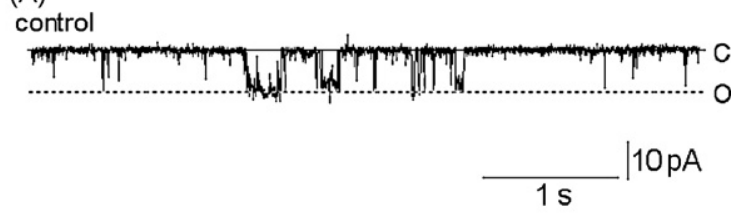

8нM hGSTM2-2
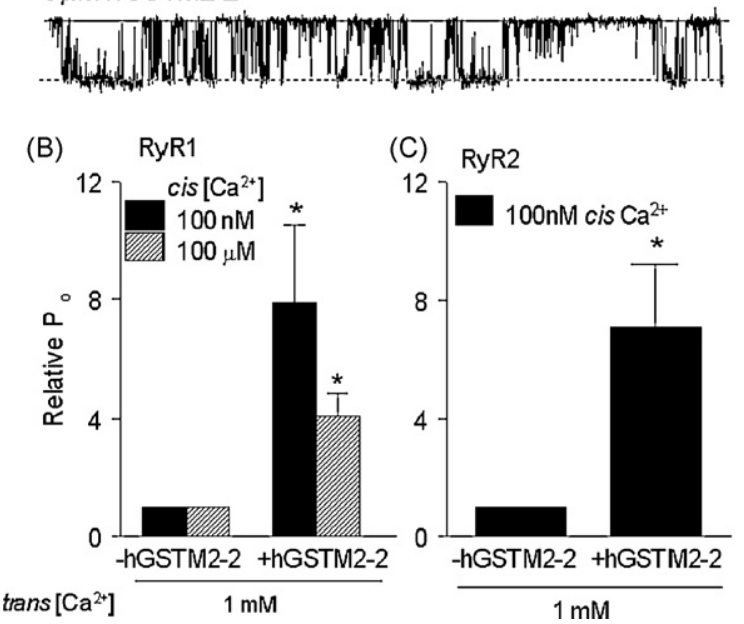

Fig. 4. Luminal hGSTM2-2 activates RyR1 and RyR2 with $1 \mathrm{mM}$ trans $\left[\mathrm{Ca}^{2+}\right]$. (A) RyR1 channel, $-40 \mathrm{mV}, 100 \mu \mathrm{M}$ cis $\mathrm{Ca}^{2+}$; before (control) and after exposure to $8 \mu \mathrm{M}$ hGSTM2-2. Channel opening is downward from the closed level (c) to the maximum channel conductance (o). (B) and (C) Average data for RyR1 (A) exposed to 8-20 $\mu$ M hGSTM2-2 (100 $\mathrm{nM}$ cis $\mathrm{Ca}^{2+}, n=16$; filled bins or $100 \mu \mathrm{M}$ cis $\mathrm{Ca}^{2+} n=12$; hatched bins) or RyR2 (C, $100 \mathrm{nM}$ cis $\mathrm{Ca}^{2+}, n=8$ ) with $20 \mu \mathrm{M}$ hGSTM2-2. Data at $+40 \mathrm{mV}$ and $-40 \mathrm{mV}$ is included in the averages. The asterisk (*) indicates a significant difference from control.

tion from the cis chamber (Ahern et al., 1994; Laver et al., 1995; Wei et al., 2006). Because of the usual scatter in absolute $P_{\mathrm{o}}$ values between individual experiments (e.g. 0.0001-0.05 in RyR1 with $100 \mathrm{nM}$ cis $\mathrm{Ca}^{2+}$ ), data is expressed as relative $P_{\mathrm{o}}$ calculated for each individual experiments, where relative $P_{\mathrm{o}}$ is $P_{\mathrm{o}}$ in the presence of GSTM2-2 and/or GSTM2-2 plus BAPTA relative to $P_{\mathrm{o}}$ under control conditions. The increase in relative $P_{\mathrm{o}}$ with hGSTM2-2 was similar and significant in RyR1 and RyR2 channels when the cytoplasmic (cis) $\mathrm{Ca}^{2+}$ concentration was $100 \mathrm{nM}$ (filled bars, Fig. 4B and C). 
Similar effects of hGSTM2-2 were observed when RyR1 channels were examined with $100 \mu \mathrm{M} \mathrm{Ca}^{2+}$ (hatched bar, Fig. 4B), indicating that effects of hGSTM2-2 were independent of cytoplasmic $\mathrm{Ca}^{2+}$ concentration.

\subsection{Effects of CSQ and hGSTM2-2 on the luminal side of RyR channels with low luminal $\left[\mathrm{Ca}^{2+}\right]$ $(<1 \mathrm{mM})$}

RyR channels associated with CSQ monomers (rather than polymers) can be examined using luminal $\left[\mathrm{Ca}^{2+}\right]$ in the range of $100 \mathrm{nM}$ to $100 \mu \mathrm{M}$. Several minutes exposure to a $\left[\mathrm{Ca}^{2+}\right] \leq 100 \mu \mathrm{M}$ leads to depolymerisation, while leaving the terminal endogenous CSQ monomersbound triadin and junctin (Wei et al., 2006) which is stronger at lower $\left[\mathrm{Ca}^{2+}\right]$ (Shin, Ma, \& Kim, 2000). Depolymerisation is demonstrated with chemical crosslinking and by a reduction in CSQ associated with the RyR (Wei et al., 2006). Addition of hGSTM2-2 to the low luminal $\mathrm{Ca}^{2+}$ solution increased the activity of RyR 1 and RyR2 channels (Fig. 5A and B) and increased channel conductance (as with $1 \mathrm{mM}$ luminal $\mathrm{Ca}^{2+}$ (Fig. 3C and $\mathrm{D}$ above)), indicating that the hGSTM2-2-induced effects were also largely independent of luminal $\left[\mathrm{Ca}^{2+}\right]$.

The effect of adding CSQ to RyRs maintained in low $\left[\mathrm{Ca}^{2+}\right]$ had not been determined. CSQ at $0.4 \mu \mathrm{M}$ $(20 \mu \mathrm{g} / \mathrm{ml})$ produces maximal effects on RyRs (Beard et al., 2002) and increased both RyR1 and RyR2 activity (Fig. 5C and D). Notably channel conductance was unaffected (see Fig. 6A below). Since CSQ does not selfassociate (dimerise or polymerise) when the $\left[\mathrm{Ca}^{2+}\right]$ is $\leq 100 \mu \mathrm{M}$ (Wei et al., 2006), the added CSQ monomers would not have associated with residual CSQ monomers with the triadin/junctin RyR complex. The added CSQ may however have interacted directly with the RyR (which increases RyR1 activity (Beard et al., 2002; Szegedi et al., 1999)). Alternatively, CSQ could have associated with a different protein in the RyR complex. When the luminal $\mathrm{Ca}^{2+}$ bathing the residual CSQ monomer/triadin/junctin/RyR1 complex is raised from $\leq 100 \mu \mathrm{M}$ to $1 \mathrm{mM}$, adding exogenous CSQ decreases RyR channel activity (Wei et al., 2006). Thus, in contrast to hGSTM2-2, the effect of CSQ on the luminal side of the RyR is luminal $\left[\mathrm{Ca}^{2+}\right]$-dependent.

\subsection{Reversibility of luminal actions of hGSTM2-2}

GSTs could alter RyR activity by ligand binding, or redox-sensor effects which are not mediated by the formation of disulphide bonds (Feng, Liu, Allen, \& Pessah, 2000) or by the formation of disulphide bonds. Ligand binding and redox-sensor effects can be reversed simply by washing the oxidant or protein out of the bath, while the reduction of disulphide bonds cannot be achieved by washout. In 5 experiments on RyR2 channels, with $100 \mathrm{nM}$ cis $\mathrm{Ca}^{2+}$ and $1 \mathrm{mM}$ trans (luminal) $\mathrm{Ca}^{2+}$, luminal hGSTM2-2 caused a significant $1.75 \pm 0.43$-fold increase in open probability which fell to $1.03 \pm 0.31$ when the luminal chamber was perfused to remove the protein. The conductance increased $1.29 \pm 0.13$-fold when hGSTM2-2 was added and then fell to $0.98 \pm 0.23$ of control after washout. The changes in open probability and conductance occurred simultaneously, indicating that the two effects could be the result of one process. The reversibility indicates that hGSTM2-2 acts through ligand binding or other redox-sensor mechanisms which could potentially occur between $\mathrm{CH}_{2} \mathrm{SH}$ groups in hGSTM2-2 and on the luminal domains of the RyR or its associated proteins (Feng et al., 2000), rather than by disulphide formation.

The effect of RyR oxidation state on the luminal actions of hGSTM2-2 and CSQ were indirectly assessed by examining the degree of activation by the luminal hGSTM2-2 and CSQ as a function of their initial open probability under control conditions (Fig. 5E and F). It is well established that RyR channel activity is strongly dependent on the number of free -SH groups in the protein, which can vary between $\sim 12$ and 50 per subunit (Marengo, Hidalgo, \& Bull, 1998; Sun, Xu, Eu, Stamler, $\&$ Meissner, 2001). It is apparent in Fig. 5E that larger increases in activity with GSTM2-2 occur in channels with an initial open probability less than 0.01 . There was effectively no potentiation by hGSTM2-2 in channels with initial open probabilities greater than 0.1. In contrast, with CSQ (Fig. 5F), larger increases in activity can be seen over the full range initial open probabilities, although simple regression analysis did not yield a strong correlation between activity increase and initial open probability in either case. The confinement of strong effects of GST to channels with low activity could indicate that free $-\mathrm{SH}$ groups were a requirement for the GST interaction and that there was an interaction between hGSTM2-2 and redox sensors in the luminal domain of the RyRs (Feng et al., 2000). The preferential activation of low activity channels by GST and not by CSQ provides a further evidence of the differential actions of the two proteins.

\subsection{Increase in conductance with luminal hGSTM2-2}

This increase in conductance was seen under all conditions (Fig. 6). The conductance also increased 
(A)

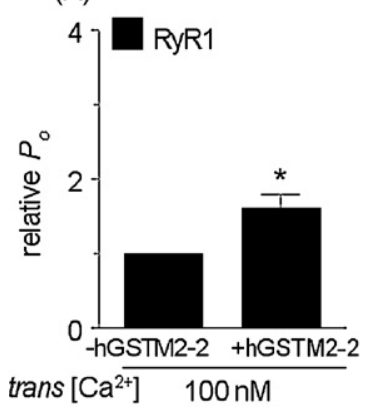

(E)
(B)

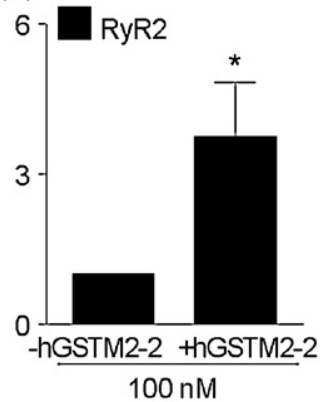

(C)



(D)

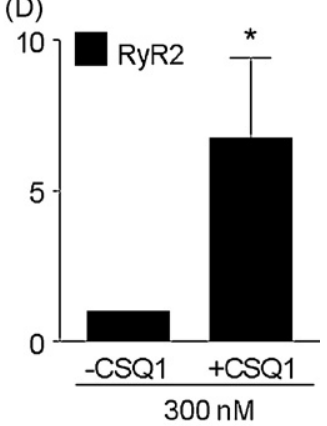

(F)

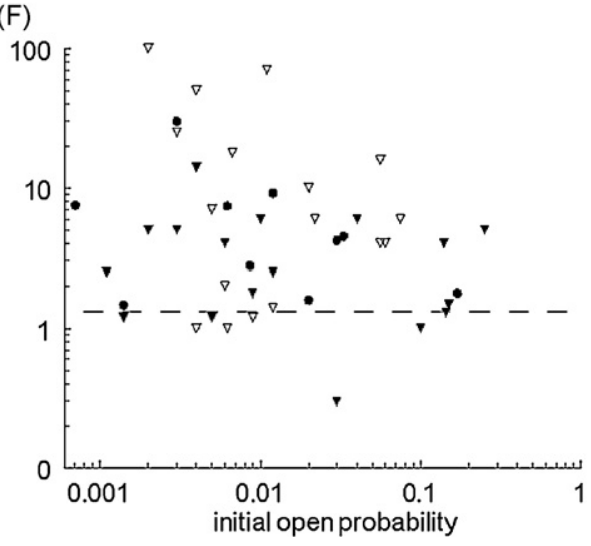

Fig. 5. hGSTM2-2 (8-30 $\mu \mathrm{M})$ and CSQ $(0.4 \mu \mathrm{M})$ in the luminal solution activate RyR1 and RyR2 when the luminal [Ca $\left.{ }^{2+}\right]$ is $100-300 \mathrm{nM}$ and RyR activity dependence of hGSTM2-2 and CSQ1. (A) trans CSQ1 on RyR1 with $100 \mathrm{nM}$ cis Ca ${ }^{2+}(n=16)$; (B) trans CSQ1 on RyR2 with 500 nM cis $\mathrm{Ca}^{2+}(n=24)$. (C) trans hGSTM2-2 on RyR1 with $500 \mathrm{nM}$ cis $\mathrm{Ca}^{2+}(n=10)$; (D) trans hGSTM2-2 on RyR2 $(n=14)$. The average in (D) includes data for $100 \mathrm{nM}$ and $100 \mu \mathrm{M}$ cis $\mathrm{Ca}^{2+}$ since results were similar at both $\mathrm{Ca}^{2+}$ concentrations. Data at +40 and $-40 \mathrm{mV}$ is combined. $(\mathrm{E})$ and (F) The increase in activity upon addition of hGSTM2-2 (E) or CSQ (F) to the trans chamber is plotted against the initial open probability $\left(P_{\mathrm{o}}\right)$ of the channel which is indicative of oxidation state (see text description). Filled circles: skeletal RyR1; open circles in (E): cardiac RyR2 with $1 \mathrm{mM}$ trans $\mathrm{Ca}^{2+}$. Open triangles: cardiac RyR2 with $100 \mathrm{nM}$ trans $\mathrm{Ca}^{2+}$; filled triangles in $(\mathrm{F})$ : cardiac RyR2 with $300 \mathrm{nM}$ trans $\mathrm{Ca}^{2+}$. It should be noted that "initial open probability $\left(P_{\mathrm{o}}\right)$ " was recorded with $1 \mathrm{mM}$ trans $\mathrm{Ca}^{2+}$ and $100-500 \mathrm{nM}$ cis $\mathrm{Ca}^{2+}$ before addition of BAPTA to lower luminal Ca ${ }^{2+}$ in the trans chamber in some experiments. For those experiments, $P_{\mathrm{o}}$ with hGSTM2-2 or CSQ is expressed relative to $P_{\mathrm{o}}$ in BAPTA before hGSTM2-2 or CSQ addition. Since $P_{\mathrm{o}}$ was reduced by BAPTA, the relative increase, particularly with CSQ in (F) was greater than it would have been if $P_{\mathrm{o}}$ had been expressed relative to the initial $P_{\mathrm{o}}$.

when luminal $\mathrm{Ca}^{2+}$ was dropped from $1 \mathrm{mM}$ to $18 \mu \mathrm{M}$ (Fig. 6B) due to removal of $\mathrm{Ca}^{2+}$ ions that compete with $\mathrm{Cs}^{+}$and bind to and block the channel pore (Ching, Williams, \& Sitsapesan, 2000). An additional increase in conductance occurred when GSTM2-2 was added (Fig. 6B). In contrast, CSQ1 did not increase the conductance (Fig. 6A). The change in conductance with lowering luminal $\left[\mathrm{Ca}^{2+}\right]$ is independent of voltage (Fig. 7A and B; Ching et al., 2000), as is the increase due to luminal hGSTM2-2. The conductance increase was also independent of the cis or trans $\left[\mathrm{Ca}^{2+}\right]($ Fig. $7 \mathrm{C}-\mathrm{H})$ or the presence of cis ATP (not shown).

The increase in conductance cannot be attributed to hGSTM2-2 entering the membrane which would be equally effective from the cytoplasmic or the luminal side; there was no change in conductance with cytoplas- mic application (Abdellatif et al., 2007). There was no change in baseline current with luminal hGTSM2-2 and no additional channel activity that could be attributed to channels formed by the protein. The reversibility also suggests that hGSTM2-2 does not form a channel, since channels are not removed from the bilayer by perfusion. Finally, all channel activity after addition of hGSTM22 was abolished by ruthenium red which specifically blocks RyR channels.

\subsection{Cytoplasmic addition of CSQ}

A further difference between CSQ and hGSTM22 was seen when cytoplasmic CSQ was ineffective in changing RyR activity (Fig. 8). This is in contrast to cytoplasmic hGSTM2-2 which strongly inhibits 

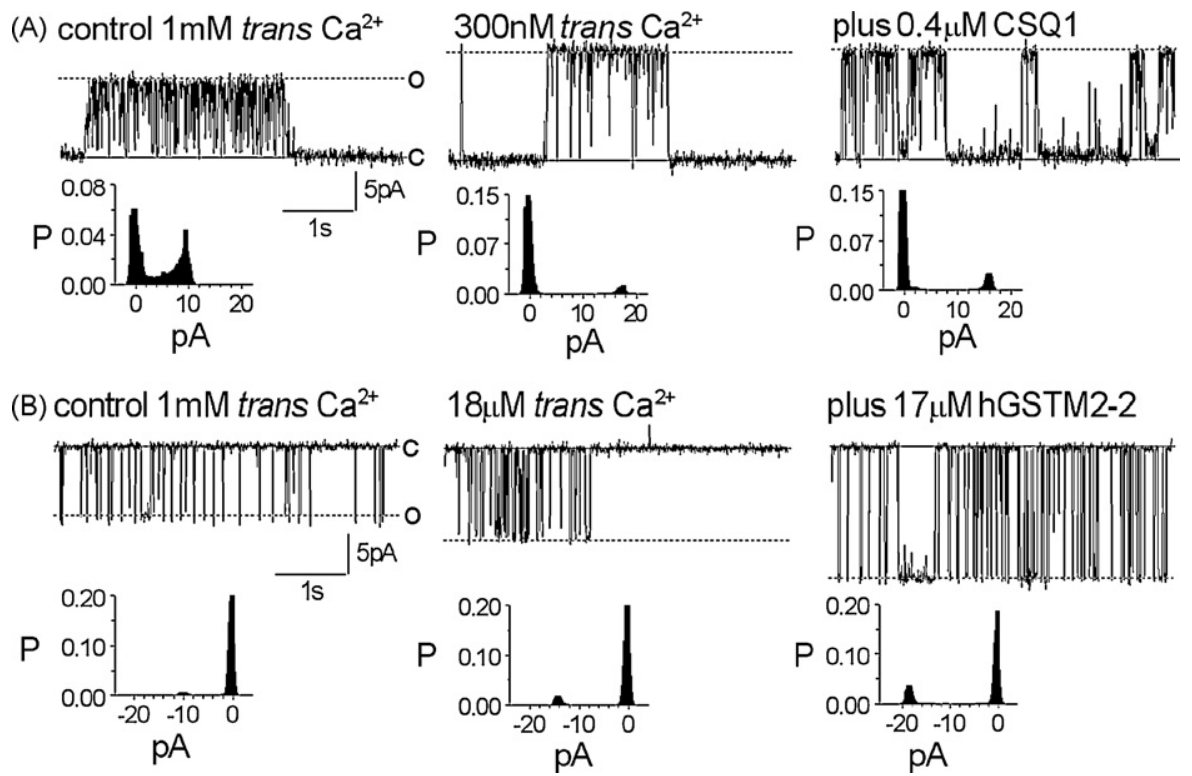

Fig. 6. hGSTM2-2, but not CSQ, alters RyR conductance. (A) RyR2 at $+40 \mathrm{mV}$ with $1 \mathrm{mM}$ trans $\mathrm{Ca}^{2+}$ (left), exposed to $300 \mathrm{nM}$ trans $\mathrm{Ca}^{2+}$ (centre) and then $0.4 \mu \mathrm{M}$ CSQ (right). The usual increase in conductance is seen when trans $\mathrm{Ca}^{2+}$ is reduced below $1 \mathrm{mM}$ (Wei et al., 2006), but there is no further conductance change with CSQ. (B) A different RyR2 channel at $-40 \mathrm{mV}$ with $1 \mathrm{mM}$ trans $\mathrm{Ca}^{2+}$ (left), then $18 \mu \mathrm{M}$ trans $\mathrm{Ca}^{2+}$ (centre), then $18 \mu \mathrm{M}$ trans $\mathrm{Ca}^{2+}$ plus $17 \mu \mathrm{M}$ trans GSTM2-2. Channel opening is upward (A), or downward (B), from the closed level (c) to the maximum open conductance (o). All-points histograms for $30 \mathrm{~s}$ of activity shown beneath each record.

RyR2 and has a voltage-dependent action on RyR1 (Abdellatif et al., 2007), with inhibition at $+40 \mathrm{mV}$ or activation $-40 \mathrm{mV}$ (Fig. 8). Thus, the actions of CSQ and hGSTM2-2 are distinctly different on the cytoplasmic as well as on the luminal side of the RyR complex.

\section{Discussion}

We provide the first evidence that an hGSTM2-2-like protein is contained within the SR in cardiac muscle. hGSTM2-2 interacts with the luminal side of the RyR complex and modifies the conductance and gating of the channel. These effects of hGSTM2-2 are distinct from the effects of CSQ, although both proteins contain thioredoxin-fold structures. This suggests that the actions of the proteins on the RyR are not mediated by the thioredoxin-fold structure alone. The amino acid sequences or structure of other regions of the proteins must determine their calcium binding capacity and glutathione transferase activity, and their interactions with the RyR complex. It is notable that the cytoplasmic actions of other members of the GST family (e.g. CLIC proteins) with weak glutathione transferase activity modify RyR in a similar manner to hGSTM2-2 (Board et al., 2004).

\subsection{GSTM2-2 in muscle interacts with RyRs}

GSTM2-2 is found in the cytoplasm of skeletal and cardiac muscle at $\sim 9-23 \mu \mathrm{M}$ (Abdellatif et al., 2007). Weak GST activity of heavy SR membranes could be due to GSTs in the lumen of the SR or associated with the SR membrane (Abdellatif et al., 2007). The present study suggests that at least some of this GST activity can be attributed to the GSTM2-2-like protein within the cardiac SR lumen or other GST family members in skeletal SR. The high concentrations of GSTs required to regulate RyRs and the rapid reversibility of their effects suggest that the interactions with the RyR are weak. However, the effects on channel activity and preliminary crosslinking data (data to be published separately) show that hGSTM2-2 can interact with the RyR2 complex.

\section{2. hGSTM2-2 interacts with the luminal domain of the RyR}

The effect of hGSTM2-2 in the luminal solution differed from its actions on the cytoplasmic side of the RyR (Fig. 8; Abdellatif et al., 2007). The $\sim 7$-fold increase in RyR2 activity with luminal application compares with the $\sim 3$-fold decrease with cytoplasmic application (Fig. 4; Abdellatif et al., 2007). Channel activation of 



Fig. 7. GSTM2-2 in the luminal solution increases RyR conductance. (A) and (B) Average current/voltage curves. Open symbols: $1 \mathrm{mM}$ trans $\mathrm{Ca}^{2+}$; filled symbols: $300 \mathrm{nM}$ trans $\mathrm{Ca}^{2+}$. Circles: before GSTM2-2; triangles: with 8-30 $\mu$ M GSTM2-2. The legend applies to (A) and (B). (C)-(H) Average conductance. (C) RyR1, $1 \mathrm{mM}$ trans $\mathrm{Ca}^{2+}, 100 \mu \mathrm{M}$ cis $\mathrm{Ca}^{2+}(n=12)$. (D) RyR2, $1 \mathrm{mM}$ trans $\mathrm{Ca}^{2+}, 100 \mathrm{nM}$ cis $\mathrm{Ca}^{2+}(n=4)$. (E) RyR1s exposed sequentially to $1 \mathrm{mM}$ trans $\mathrm{Ca}^{2+}, 18 \mu \mathrm{M}$ trans $\mathrm{Ca}^{2+}, 300 \mathrm{nM}$ trans $\mathrm{Ca}^{2+}$ and $20-30 \mu \mathrm{M}$ GSTM2-2, 100 nM cis Ca ${ }^{2+}$ ( $\left.n=14\right)$. (F) RyR2s exposed sequentially to $1 \mathrm{mM}$ trans $\mathrm{Ca}^{2+}, 18 \mu \mathrm{M}$ trans $\mathrm{Ca}^{2+}$ and then 20-30 $\mu \mathrm{M}$ GSTM2-2, $100 \mu \mathrm{M}$ cis $\mathrm{Ca}^{2+}(n=6)$. (G) RyR1 with GSTM2-2 at $1 \mathrm{mM}$ or 300 nM trans Ca ${ }^{2+}$. (H) RyR2s exposed sequentially to $1 \mathrm{mM}$ trans $\mathrm{Ca}^{2+}, 18 \mu \mathrm{M}$ trans $\mathrm{Ca}^{2+}, 300 \mathrm{nM}$ trans $\mathrm{Ca}^{2+}$ and then GSTM2-2 (at 20 or $\left.30 \mu \mathrm{M}\right), 100 \mu \mathrm{M}$ cis $\mathrm{Ca}^{2+}(n=12)$. The asterisk (*) indicates a significant difference from control. 

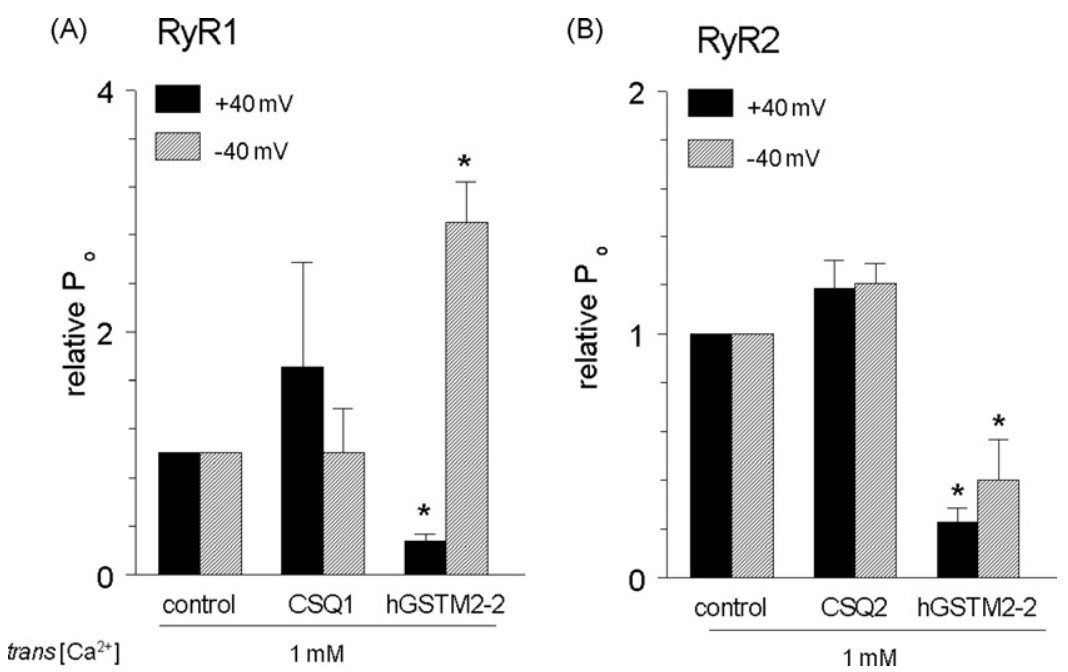

Fig. 8. CSQ and GSTM2-2 on the cytoplasmic side of RyR channels. Average relative open probability $\left(P_{\mathrm{o}}\right)(\mathrm{mean} \pm \mathrm{S}$.E.M.) for: (A) $0.4 \mu \mathrm{M}$ CSQ1 added RyR1 with a cis $\mathrm{Ca}^{2+}$ of $100 \mu \mathrm{M}(n=5)$; (B) $0.4 \mu \mathrm{M}$ CSQ2 added to RyR2 with a cis $\mathrm{Ca}^{2+}$ of $300 \mathrm{nM}(n=6)$. Data for GSTM2-2 from (Abdellatif et al., 2007) with $100 \mu \mathrm{M}$ cis $\mathrm{Ca}^{2+}$ for both RyR1 and RyR2, included for comparison. Filled bins: $+40 \mathrm{mV}$; cross-hatched bins: $-40 \mathrm{mV}$. The trans $\mathrm{Ca}^{2+}$ was $1 \mathrm{mM}$ in all experiments.

RyR1 occurs with both luminal and cytoplasmic addition to skeletal channels, but there is no change in conductance with cytoplasmic activation (Abdellatif et al., 2007). The data indicates that hGSTM2-2 binds to specific (and obviously different) sites on the cytoplasmic and luminal sides of the RyR. Our conclusion is that the cytoplasmic effects of hGSTM2-2, like those of DIDS (Hill \& Sitsapesan, 2002) depend on cytoplasmic hGSTM2-2 binding having a long range allosteric effect on voltage-sensors within the molecule. Since binding to the luminal side of the RyR does not alter voltagedependent inactivation the luminal binding site is not linked to the voltage-sensors that dictate inactivation.

\subsection{The location of the $h G S T M 2-2$ and CSQ luminal binding sites}

It is unlikely that hGSTM2-2 binds to the CSQ binding site on triadin or junctin since CSQ was associated with the anchoring proteins under all conditions (Shin et al., 2000; Wei et al., 2006). We cannot exclude the possibility that hGSTM2-2 binds to other sites on triadin/junctin or that some triadin/junctin sites lacked CSQ and that hGSTM2-2 modifies activity by binding to those sites. The specific action of luminal hGSTM2-2 on conductance argues for separate CSQ and hGSTM2-2 binding sites (model below).

The maintained association between the endogenous CSQ with the triadin/junctin/RyR complex under all conditions suggests that the triadin/junctin binding sites were not available for additional CSQ binding. Exogenous CSQ could bind to sites located either on the luminal domains of the RyR or other proteins associated with the luminal side of the RyR. Direct binding of CSQ1 to purified RyR1 increase channel activity (Beard et al., 2002; Szegedi et al., 1999), as does CSQ addition to RyRs with low luminal $\left[\mathrm{Ca}^{2+}\right]$ (Fig. 6A). Thus, these effects could well be due to direct binding.

\subsection{A model for the effects of hGSTM2-2 on RyR conductance}

The change in conductance with luminal hGSTM22 reveals a novel biophysical/biochemical property of GSTs. This is the first report of a protein-protein interaction increasing the maximum channel conductance. Conductance is an intrinsic property of the pore and considered to be influenced by factors that interact with the pore. For example, ion species (Ching et al., 2000), compounds that enter and block the pore, residues that line the pore and/or residues that form the selectivity filter. Since voltage-dependent effects of luminal hGSTM2-2 were not observed, it is likely hGSTM2-2 binds outside the membrane field, but close to the pore where it could influence conductance, perhaps binding to the selectivity filter region. hGSTM2-2 has regions with positive charge density (Fig. 3) which could bind to negatively charged residues in the selectivity filter. There are also surfaces of negative charge density on the monomer. Thus one hypothesis is that: 


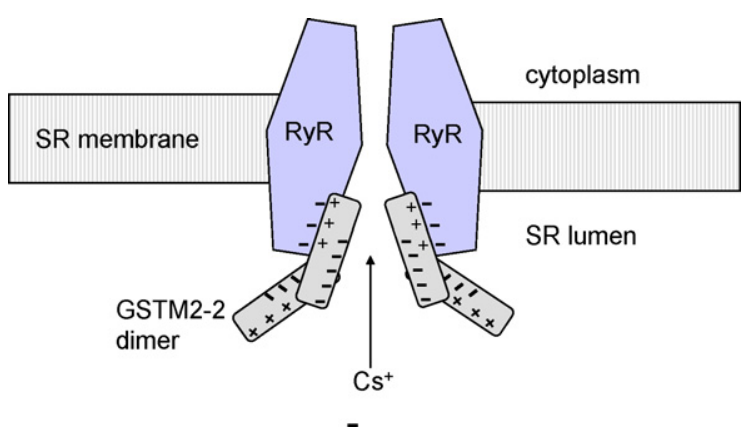

Fig. 9. A model for the increase in conductance when GSTM2-2 dimer interacts with the luminal domain of RyR channels. A positively charged surface of GSTM2-2, as on the surface of the thioredoxin or helical domain (Fig. 2) could interact with negatively charged residues near the channel pore. Negatively charged residues on the opposite surface of four GSTM2-2 molecules (one bound to each RyR subunit) would line a pseudo pore formed by the 4 GSTM2-2 entities. Two of the four subunits of the RyR are shown.

(a) a positive surface on hGSTM2-2 binds to acidic residues on the pore, with one GST dimer binding per RyR monomer (Fig. 9).

(b) The negative charges on non-binding side of the hGSTM2-2 dimer then form a quasi-pore between hGSTM2-2 molecules bound to each of the four subunits.

(c) A higher negative charge density on the hGSTM2-2 would facilitate a higher channel conductance.

This hypothesis could be tested in the future by mutating acidic residues in GSTM2-2.

\subsection{Role of GSTM2-2 in the SR lumen}

GSTM2-2 may access the lumen of the cardiac SR by diffusing through the SR membrane or by a transport process. GSTs have the potential to penetrate and interact with membranes (Merezhinskaya, Kuijpers, \& Raviv, 1998; Namiki, Tomida, Tanabe, Iino, \& Hirose, 2003), but it is not clear whether this is an active or a passive process. CLIC members of the GST structural family (Dulhunty et al., 2001) exist as soluble proteins or as membrane-bound ion channels (Cromer, Morton, Board, \& Parker, 2002; Harrop et al., 2001; Tulk, Schlesinger, Kapadia, \& Edwards, 2000) and thus have the capacity to pass through the membrane environment into the SR lumen. The fact that the GSTM2-2-like protein is in cardiac, but not in skeletal SR, suggests that a specific GSTM2-2 uptake mechanism is located only in the cardiac SR membrane.

GSTM2-2 in SR would enhance $\mathrm{Ca}^{2+}$ fluxes through the RyR. This would counteract the inhibitory cyto- plasmic effects of GSTM2-2 on RyR2. If GST entry into the SR lumen is ATP-dependent, less may enter in fatigued muscle and the RyR-activating luminal actions of GSTM2-2 be reduced to protect $\mathrm{Ca}^{2+}$ within the store. GSTM2-2 in the SR may also, by virtue of its glutathione transferase activity, play a house keeping role by conjugating GSH to any electrophilic compounds that accumulate within the SR.

In conclusion, we provide the first evidence for the presence of a GST in the lumen of an internal membrane system. We show a soluble hGSTM2-2-like protein exists within the SR of cardiac muscle and show that luminal hGSTM2-2 is a novel luminal regulator of RyR2 and RyR1. The results raise the possibility that GSTs form a class luminal regulators of proteins located in ER or SR membranes and that the type of GST in the lumen of the SR/ER is tissue specific.

\section{Acknowledgements}

We thank Suzy Pace and Joan Stivala for SR vesicle preparations and Marco Casarotto and Nicole Norris for assistance with the PyMol program. The work was supported by grants from the Australian NH\&MRC \#268027 and \#471462.

\section{References}

Abdellatif, Y., Liu, D., Gallant, E. M., Gage, P. W., Board, P. G., \& Dulhunty, A. F. (2007). The Mu class glutathione transferase is abundant in striated muscle and is an isoform-specific regulator of ryanodine receptor calcium channels. Cell Calcium, 41, 429-440.

Ahern, G. P., Junankar, P. R., \& Dulhunty, A. F. (1994). Single channel activity of the ryanodine receptor calcium release channel is modulated by FK-506. FEBS Letters, 352, 369-374.

Beard, N. A., Casarotto, M. G., Wei, L., Varsanyi, M., Laver, D. R., \& Dulhunty, A. F. (2005). Regulation of ryanodine receptors by calsequestrin: Effect of high luminal $\mathrm{Ca}^{2+}$ and phosphorylation. The Biophysical Journal, 88, 3444-3454.

Beard, N. A., Laver, D. R., \& Dulhunty, A. F. (2004). Calsequestrin and the calcium release channel of skeletal and cardiac muscle. Progress in Biophysics and Molecular Biology, 85, 33-69.

Beard, N. A., Sakowska, M. M., Dulhunty, A. F., \& Laver, D. R. (2002). Calsequestrin is an inhibitor of skeletal muscle ryanodine receptor calcium release channels. The Biophysical Journal, 82, 310-320.

Board, P. G., Coggan, M., Chelvanayagam, G., Easteal, S., Jermiin, L. S., Schulte, G. K., et al. (2000). Identification, characterization, and crystal structure of the Omega class glutathione transferases. The Journal of Biological Chemistry, 275, 24798-24806.

Board, P. G., Coggan, M., Watson, S., Gage, P. W., \& Dulhunty, A. F. (2004). CLIC-2 modulates cardiac ryanodine receptor $\mathrm{Ca}(2+)$ release channels. The International Journal of Biochemistry and Cell Biology, 36, 1599-1612.

Board, P. G., Suzuki, T., \& Shaw, D. C. (1988). Human muscle glutathione $S$-transferase (GST-4) shows close homology to human liver GST-1. Biochemica et Biophysica Acta, 953, 214-217. 
Campbell, K. P., MacLennan, D. H., \& Jorgensen, A. O. (1983). Staining of the $\mathrm{Ca}^{2+}$-binding proteins, calsequestrin, calmodulin, troponin C, and S-100, with the cationic carbocyanine dye "Stainsall". The Journal of Biological Chemistry, 258, 11267-11273.

Chamberlain, B. K., \& Fleischer, S. (1988). Isolation of canine cardiac sarcoplasmic reticulum. Methods in Enzymology, 157, 91-99.

Ching, L. L., Williams, A. J., \& Sitsapesan, R. (2000). Evidence for $\mathrm{Ca}^{2+}$ activation and inactivation sites on the luminal side of the cardiac ryanodine receptor complex. Circulation Research, 87, 201-206.

Cromer, B. A., Morton, C. J., Board, P. G., \& Parker, M. W. (2002). From glutathione transferase to pore in a CLIC. The European Biophysical Journal, 31, 356-364.

Dulhunty, A., Gage, P., Curtis, S., Chelvanayagam, G., \& Board, P. (2001). The glutathione transferase structural family includes a nuclear chloride channel and a ryanodine receptor calcium release channel modulator. The Journal of Biological Chemistry, 276, 3319-3323.

Dulhunty, A. F., Pouliquin, P., Coggan, M., Gage, P. W., \& Board, P. G. (2005). A recently identified member of the glutathione transferase structural family modifies cardiac RyR2 substate activity, coupled gating and activation by Ca2+ and ATP. Biochemical Journal, 390, 333-343.

Feng, W., Liu, G., Allen, P. D., \& Pessah, I. N. (2000). Transmembrane redox sensor of ryanodine receptor complex. The Journal of Biological Chemistry, 275, 35902-35907.

Harrop, S. J., DeMaere, M. Z., Fairlie, W. D., Reztsova, T., Valenzuela, S. M., Mazzanti, M., et al. (2001). Crystal structure of a soluble form of the intracellular chloride ion channel CLIC1 (NCC27) at 1.4-A resolution. The Journal of Biological Chemistry, 276, 44993-45000.

Herzog, A., Szegedi, C., Jona, I., Herberg, F. W., \& Varsanyi, M. (2000). Surface plasmon resonance studies prove the interaction of skeletal muscle sarcoplasmic reticular $\mathrm{Ca}(2+)$ release channel/ryanodine receptor with calsequestrin. FEBS Letters, 472, 73-77.

Hill, A. P., \& Sitsapesan, R. (2002). DIDS modifies the conductance, gating, and inactivation mechanisms of the cardiac ryanodine receptor. The Biophysical Journal, 82, 3037-3047.

Kim, D. H., Ohnishi, S. T., \& Ikemoto, N. (1983). Kinetic studies of calcium release from sarcoplasmic reticulum in vitro. The Journal of Biological Chemistry, 258, 9662-9668.

Laemmli, U. K. (1970). Cleavage of structural proteins during the assembly of the head of bacteriophage T4. Nature, 227, 680-685.

Laver, D. R., Roden, L. D., Ahern, G. P., Eager, K. R., Junankar, P. R., \& Dulhunty, A. F. (1995). Cytoplasmic $\mathrm{Ca}^{2+}$ inhibits the ryanodine receptor from cardiac muscle. The Journal of Membrane Biology, 147, 7-22.

Mannervik, B., Awasthi, Y. C., Board, P. G., Hayes, J. D., Di Ilio, C., Ketterer, B., et al. (1992). Nomenclature for human glutathione transferases. Biochemical Journal, 282(Pt 1), 305-306.
Marengo, J. J., Hidalgo, C., \& Bull, R. (1998). Sulfhydryl oxidation modifies the calcium dependence of ryanodine-sensitive calcium channels of excitable cells. The Biophysical Journal, 74, 1263-1277.

Maruyama, K., Mikawa, T., \& Ebashi, S. (1984). Detection of calcium binding proteins by $45 \mathrm{Ca}$ autoradiography on nitrocellulose membrane after sodium dodecyl sulfate gel electrophoresis. The Journal of Biochemistry (Tokyo), 95, 511-519.

Merezhinskaya, N., Kuijpers, G. A., \& Raviv, Y. (1998). Reversible penetration of alpha-glutathione $S$-transferase into biological membranes revealed by photosensitized labelling in situ. Biochemical Journal, 335(Pt 3), 597-604.

Namiki, S., Tomida, T., Tanabe, M., Iino, M., \& Hirose, K. (2003). Intracellular delivery of glutathione $S$-transferase into mammalian cells. Biochemical Biophysical Research Communications, 305, 592-597.

Patskovska, L. N., Fedorov, A. A., Patskovsky, Y. V., Almo, S. C., \& Listowsky, I. (1998). Expression, crystallization and preliminary X-ray analysis of ligand-free human glutathione $S$-transferase M2-2. Acta Crystallography D Biological Crystallography, 54, $458-460$.

Ross, V. L., \& Board, P. G. (1993). Molecular cloning and heterologous expression of an alternatively spliced human Mu class glutathione S-transferase transcript. Biochemical Journal, 294(Pt 2), 373380.

Saito, A., Seiler, S., Chu, A., \& Fleischer, S. (1984). Preparation and morphology of sarcoplasmic reticulum terminal cisternae from rabbit skeletal muscle. The Journal of Cell Biology, 99, 875885.

Shin, D. W., Ma, J., \& Kim, D. H. (2000). The asp-rich region at the carboxyl-terminus of calsequestrin binds to $\mathrm{Ca}(2+)$ and interacts with triadin. FEBS Letters, 486, 178-182.

Sun, J., Xu, L., Eu, J. P., Stamler, J. S., \& Meissner, G. (2001). Classes of thiols that influence the activity of the skeletal muscle calcium release channel. The Journal of Biological Chemistry, 276, $15625-15630$.

Szegedi, C., Sarkozi, S., Herzog, A., Jona, I., \& Varsanyi, M. (1999). Calsequestrin: more than 'only' a luminal $\mathrm{Ca} 2+$ buffer inside the sarcoplasmic reticulum. Biochemical Journal, 337, 19-22.

Tulk, B. M., Schlesinger, P. H., Kapadia, S. A., \& Edwards, J. C. (2000). CLIC-1 functions as a chloride channel when expressed and purified from bacteria. The Journal of Biological Chemistry, 275, 26986-26993.

Wang, S., Trumble, W. R., Liao, H., Wesson, C. R., Dunker, A. K., \& Kang, C. H. (1998). Crystal structure of calsequestrin from rabbit skeletal muscle sarcoplasmic reticulum. Nature Structural Biology, $5,476-483$.

Wei, L., Varsanyi, M., Dulhunty, A. F., \& Beard, N. A. (2006). The conformation of calsequestrin determines its ability to regulate skeletal ryanodine receptors. The Biophysical Journal, 91, 1288-1301. 